\title{
Linfoma no Hodgkin difuso de células grandes B en estadios avanzados: situación del tratamiento actual y perspectivas
}

Durante décadas, el régimen CHOP (ciclofosfamida, adriamicina, vincristina y prednisona) cada 21 días, ha sido el tratamiento estándar del linfoma difuso de células grandes B (LDCGB). Ningún otra pauta de tercera generación demostró ser mejor ${ }^{1}$.

En el año 2002, el grupo GELA (Groupe d'Etude des Lymphomes de l'Adulte) publica los resultados de su estudio LHN98-5 en pacientes de más de 60 años con LDCGB en los que la adición de Rituximab (R) a la pauta CHOP incrementaba significativamente el número de respuestas, la supervivencia libre de enfermedad y la supervivencia global tras dos años de seguimiento ${ }^{2}$. Estos resultados se han visto confirmados posteriormente tras cinco años de seguimiento ${ }^{3}$. Actualmente, R-CHOP es el tratamiento estándar de primera línea en pacientes con LDCGB en todos los grupos de edad ${ }^{4,5}$.

La introducción de R-CHOP en la primera línea de tratamiento ha mejorado de forma espectacular el futuro de los pacientes de buen pronóstico; menos del $20 \%$ de los pacientes con bajo o moderado bajo riesgo del IPI van a presentar una recidiva. Sin embargo, más del $40 \%$ de los pacientes con riesgo alto o moderado-alto van a recaer en los primeros 4 años.

La quimioterapia intensiva en linfomas se viene desarrollando desde los años 70. El principio básico sobre el que se soporta la quimioterapia intensiva es la presunción de la existencia de una curva de dosis-respuesta, de agentes citotóxicos de corta vida media con fundamental toxicidad medular y de la posibilidad de rescate hematológico posterior. El esfuerzo por lograr mejorar los resultados en aquellos pacientes con factores de mal pronóstico, provocó la consecución de diferentes ensayos en fase II que mejoraban la supervivencia global con respecto a controles históricos ${ }^{6-8}$.

Con posterioridad, se han llevado a cabo diversos estudios aleatorios en pacientes en esta situación. Uno de los más importantes fue llevado a cabo también por el grupo GELA sin que se obtuviera beneficio en la supervivencia, salvo estudios en subgrupos denominados de intermedio y alto grado según el IPI ${ }^{9}$. Estos resultados no se vieron refrendados en otro estudio aleatorio dirigido a esa específica población de mal pronóstico ${ }^{10}$. La discusión sobre este aspecto ha continuado y se han sucedido los ensayos clínicos ${ }^{11-14}$ intentando esclarecer la situación e identificar un subgrupo que pudiera beneficiarse de las altas dosis como consolidación de la primera remisión. Así el grupo GOELAMS identifica a los pacientes con un IPI intermedio-alto como posibles beneficiarios de una quimioterapia intensiva en primera línea de tratamiento ${ }^{15}$. La controversia motivó un metaanálisis ${ }^{16}$ de todos los ensayos clínicos, prospectivos, aleatorios que comparaban quimioterapia estándar convencional frente a tratamiento de altas dosis en primera línea. De los 124 ensayos potencialmente analizables, sólo se analizaron 11 dado que el resto o no proporcionaban información completa o no cumplían unos criterios de inclusión aceptables. En total, se analizaron 2228 pacientes. La mortalidad global fue comparable entre ambos grupos, muriendo un 36,9\% en el grupo de quimioterapia intensiva y un 36,4\% en el grupo control. Se analizó la causa de la heterogeneidad entre grupos y sólo un tratamiento de inducción suficientemente extenso, de al menos 6 ciclos de CHOP o variantes, antes de las altas dosis, resultó significativamente mejor. A la vista de los resultados, y admitiendo la gran heterogeneidad entre los estudios, tanto metodológicos como de tratamiento, no se puede concluir que en la actualidad, exista claramente un beneficio de la quimioterapia intensiva con reinfusión de progenitores hematopoyéticos, comúnmente denominado "trasplante de médula ósea", en primera línea de tratamiento en linfomas no hodgkin difusos. Pudiera existir una población de muy alto riesgo de recaída ${ }^{17,18}$ que se beneficiara de una consolidación de altas dosis, pero siempre después de obtener una remisión completa con quimioterapia convencional ${ }^{19}$. 
A mitad de camino entre las altas dosis y el CHOP convencional, ha aparecido una tercera vía de investigación y progreso, la búsqueda de dosis densas de CHOP. Dos estudios del grupo alemán (DSHNHL) han demostrado recientemente que dosis estándar de CHOP cada 14 días mejoraban la supervivencia global con respecto a las dosis cada 21 días $^{20,21}$. El grave inconveniente a la hora de analizar estos estudios es que ninguno de ellos llevaba asociado Rituximab, sin duda el fármaco que más añade de forma individual al éxito del tratamiento. Nos quedaba conocer qué podía aportar añadir el anticuerpo monoclonal al CHOP cada 14 días. Para responder a esta cuestión el activo grupo alemán DSHNHL ha concluido recientemente el ensayo RICOVER 60 presentado recientemente como comunicación a congreso ${ }^{22}$. El ensayo aleatorizaba a 1330 pacientes entre 6-8 ciclos de CHOP con y sin rituximab cada 14 días. Con un seguimiento mediano de 26 meses, no encontraron diferencias en la supervivencia libre de fallo ni en la global entre 6 u 8 ciclos, y sí hallaron beneficio en la supervivencia libre de fallo para R-CHOP cada 14 días sobre CHOP-14, aunque no en la supervivencia global. Dado que no se contaba con una rama de R-CHOP cada 21 días, no podemos saber qué diferencias podría existir con éste. El grupo español GOTEL ${ }^{23}$ presentó en ASCO 2006, los resultados de un estudio prospectivo y multicéntrico de R-CHOP 14. Se incluyeron 80 pacientes menores de 70 años, se obtuvieron un $76 \%$ de respuestas completas. Hubo una muerte tóxica, y un $15 \%$ de infecciones grado 3/4. Con un seguimiento mediano de 26 meses, la supervivencia libre de progresión fue del $72 \%$ y la supervivencia global fue del $86 \%$ demostrando posibilidad de su administración sin importante toxicidad, y la alta tasa de respuestas con este régimen

Una comparación histórica entre el R-CHOP cada 21 días del estudio GELA LHN98-5 y el R-CHOP-14 del estudio RICOVER-60, arrojaría un beneficio de supervivencia cercano al 10\% en los pacientes con peor pronóstico (64\% vs 54\%).

Nos encontramos pendientes de los resultados definitivos de este estudio que puede hacer modificar un estándar que parecía iba a durar décadas como el CHOP de la era pre-rituximab.

\author{
M. Provencio ${ }^{1}$, A. Rueda ${ }^{2}$, P. Sabín ${ }^{3}$ \\ ${ }^{1}$ Servicio de Oncología Médica. Hospital Universitario Puerta de Hierro. Madrid \\ ${ }^{2}$ Servicio de Oncología Médica. Hospital Virgen de la Victoria. Málaga \\ ${ }^{3}$ Servicio de Oncología Médica. Hospital Universitario Gregorio Marañón. Madrid
}

\title{
Bibliografía
}

1. Fisher RI, Gaynor ER, Dahlberg S, et al. Comparison of a standard regimen (CHOP) with thre intensive chemotherapy regimens for advanced non-Hodgkin's lymphoma. N Engl J Med 1993; 328: 1002-1006.

2. Coiffier B, Lepage E, Biere J, et al. CHOP chemoterapy plus rituximab compared with CHOP alone in ederly patients with diffuse large-B-cell lymphoma. N Engl J Med 2002; 346: 235-242,.

3. Feugier P, Van Hoof A, Sebban C, et al. Long-term results of the R-CHOP study in the treatment of elderly patients with diffuse large B-cell Lymphoma: A study by the Groupe dÉtude des Lymphomes de l'Adulte. J Clin Oncol 2005; 23: 4117-26.

4. Pfreundschuh MG, Trümper L, Ma D, et al. Randomized intergroup trial of first line treatment for patients $<=60$ years with diffuse large B-cell non-Hodgkin's lymphoma (DLBCL) with a CHOP-like regimen with or without the anti-CD 20 antibody rituximab: Early stopping after the first interim analysis. Proc Am Soc Clin Oncol 2004; 23: 556. (abstr 6500).

5. Sehn LH, Donaldson J, Chhanabhai M, et al. Introduction of combined CHOP plus Rituximab therapy dramatically improved outcome of diffuse large B-cell lymphoma in Brithis Columbia. J Clin Oncol 2005; 23: 5027-5033.

6. Gulati SC, Shank B, Black P et al. Autologous bone marrow transplantation for patients with poor-prognosis lymphoma. J Clin Oncol 1988; 6:1303-1313.

7. Pettengell R, Radford JA, Morgenstern GR, et al. Survival benefif from high-dose therapy with autologous blood progenitor-cell transplantation in poor-prognosis non-Hodgkin's lymphoma. J Clin Oncol 1996; 14: 586-592.

8. Cortelazzo S, Rossi A, Bellavita P, et al. Clinical outcome after autologous transplantation in non-Hodgkin's lymphoma patients with high International Prognostic Index (IPI). Ann Oncol 1999; 10:427-432. 
9. Haioun C, Lepage E, Gisselbrecht C, et al. Survival benefit of high-dose therapy in poor-risk aggressive non-hodgkin's lymphoma: final analysis of the prospective LNH 87-2 protocol-a Groupe d'Etude des Lymphomes de l'adulte study. J Clin Oncol 2000; 18:3025-3030.

10. Gisselbrecht C, Lepage E, Molina T, et al. Shortened first-line high-dose chemotherapy for patients with poor-prognosis aggressve lymphoma. J Clin Oncol 2002; 20: 2472-2479.

11. Martelli M, Gherlinzoni F, De Renzo A, et al. Early autologous stem-cell transplantation versus conventional chemotherapy as front-line therapy in high-risk, aggressive non-Hodgkin's lymphoma: an Italian Multicentrer Randomized Trial. J Clin Oncol 2003; 21; 1255-1262.

12. Kluin-Nelemans HC, Zagonel V, Anastopoulou A, et al. Standard chemotherapy with or without high-dose chemotherapy for aggressive non-Hodgkin's lymphoma: randomised phase III study. J Natl Cancer Inst 2001; 93:22-30.

13. Gianni AM, Bregni M, Siena S, et al. High-dose chemotherapy and autologous bone marrow transplantation compared with MACOP_B in aggressive B-cell lymphoma. N Engl J Med 1997; 336: 1290-1297.

14. Santini G, Salvagno L, Leoni P, et al. VACOP-B versus VACOP-B plus autologous bone marrow transplantation for advanced diffuse non-Hodgkin's lymphoma: results of a prospective randomised trial by the Non-Hodgkin's Lymphoma Cooperative Study Group. J Clin Oncol 1998; 16: 2796-2802.

15. Milpied N, Deconinck E, Gaillard F, et al. Initial treatment of aggressive lymphoma with High-dose chemotherapy and autologous stem-cell support. 2004; 350:1287-95.

16. Strehl J, Mey U, Glasmacher A, et al. High-dose chemotherapy followed by autologous stem cell transplantation as first-line therapy in aggressive non-Hodgkin's lymphoma: a meta-analysis. Haematologica 2003; 88:1304-1315.

17. Bertz H, Zeiser R, Lange W, et al. Log-term follow-up after high-dose chemotherapy and autologous stem-cell transplantation for high-grade B-cell lymphoma suggests an improved outcome for high-risk patients with respect to the age-adjusted International Prognostic Index. Ann Oncol 2004; 15: 1419-24.

18. Van Imhoff G W, van der Holt B, MacKenzie MA, et al. Impact of three courses of intensified CHOP prior to High-dose sequential therapy followed by autologous stem-cell transplantation as first-line treatment in poor-risk, aggressive non-hodgkin's lymphoma: comparative analysis of ducth-belgian hemato-oncology cooperative group studies 27 and 40. J Clin Oncol 2005; 23: 37933801 .

19. Kaiser U, Uebelacker I, Abel, U, et al. Randomized study to evaluate the use of high-dose therapy as part of primary treatment for "aggressive "lymphoma. J Clin Oncol 2002; 20: 4413-4419.

20. Pfreundschuh M, Trümper L, Klös M, et al. Two-weekly or 3-weekly CHOP chemotherapy with or without etoposide for the treatment of young patients with good-prognosis (normal LDH) aggressive lymphomas. Results of the NHL-B1 trial of the DSHNHL. The German High-Grade Non-Hodgkin's Lymphoma Study Group. Blood 2004; 104:626-33.

21. Pfreundschuh M, Trümper L, Klös M, et al. Two-weekly or 3-weekly CHOP chemotherapy with or withuot etoposide for the treatment of young patients with good-prognosis (normal LDH) aggressive lymphomas. Results of the NHL-B2 trial of the DSHNHL. The German High-Grade Non-Hodgkin's Lymphoma Study Group. Blood 2004; 104: 634-41.

22. Pfreundschuh M, Kloess M, Scmits R, et al. Six, not eight cycles of bi-weekly CHOP with rituximab (R-CHOP-14) is the preferred treatment for elderly patients with DLBCL: results of the RICOVER -60 trial of the German High-Grade Non-Hodgkin Lymphoma Study Group. Blood 2005; 106:9a (abstract 13).

23. Rueda A, Sabín P, Rifá J, et al. R-CHOP-14 in patients with diffuse large-B-cell lymphoma younger than 70 years: A multicentric and prospective study. Proceedings ASCO 2006; vol 24, nº 188 (abstract 7592). 